\title{
Síndrome de Pandora: Fisiopatogenia e Terapêutica
}

\author{
Pandora Syndrome: Physiopathogeny and Therapeutic \\ Síndrome de Pandora: Fisiopatogenia y Terapéutica
}

Recebido: 08/06/2021 | Revisado: 08/06/2021 | Aceito: 19/06/2021 | Publicado: 23/06/2021

\author{
Glenda Roberta Freire Lima \\ ORCID: https://orcid.org/0000-0002-3867-7389 \\ Universidade Estadual do Ceará, Brasil \\ E-mail: glendaroberta.medvet@gmail.com \\ Vitória Maria Jorge de Araújo \\ ORCID: https://orcid.org/0000-0001-6489-6959 \\ Universidade Estadual do Ceará, Brasil \\ E-mail: vitoria.m.arauj@gmail.com \\ Laís Dantas Ferreira \\ ORCID: https://orcid.org/0000-0003-1287-5085 \\ Universidade Estadual do Ceará, Brasil \\ E-mail: laisdantf@gmail.com \\ Francisco Douglas Lima Anastácio \\ ORCID: https://orcid.org/0000-0001-6943-4796 \\ Universidade Estadual do Ceará, Brasil \\ E-mail: douglasanastacio7@gmail.com \\ Letícia Moura Alcântara \\ ORCID: https://orcid.org/0000-0001-7314-9613 \\ Universidade Estadual do Ceará, Brasil \\ E-mail: leticia.alcantara@aluno.uece.br \\ Anna Flávia Barbosa Sousa \\ ORCID: https://orcid.org/0000-0003-0762-0731 \\ Instituição: Universidade Estadual do Ceará, Brasil \\ E-mail: anna.barbosa@aluno.uece.br \\ Nathália Ferreira Carneiro \\ ORCID: https://orcid.org/0000-0002-5154-6893 \\ Universidade Estadual do Ceará, Brasil \\ E-mail: natferreiravet@gmail.com \\ Victor Hugo Vieira Rodrigues \\ ORCID: https://orcid.org/0000-0003-2974-8015 \\ Centro Universitário Maurício de Nassau, Brasil \\ E-mail: victorvieira81@gmail.com
}

\begin{abstract}
Resumo
A Síndrome de Pandora consiste em uma desordem no trato urinário inferior de felinos, sua patogenia não está totalmente elucidada, mas sabe-se que há fatores predisponentes ao seu surgimento, como: genético, baixa taxa de ingestão hídrica, estresse ambiental, obesidade, vida doméstica moderna do gato e falta de atividades físicas. $\mathrm{O}$ objetivo deste trabalho foi relatar as principais informações descritas sobre essa afecção, além de seu impacto no organismo nestes animais. Assim, foram pesquisados artigos em bases de indexação de documentos, artigos e livros, como Scielo, Google Scholar, Pub Med e Scopus nos últimos 11 anos buscando informações mais relevantes e recentes sobre a doença. A síndrome se mostra de forma complexa que apresenta sinais clínicos inespecíficos, tornando seu diagnóstico bastante dificultoso, sendo necessário o emprego de vários exames laboratoriais. $\mathrm{O}$ tratamento envolve terapia multimodal com manejo ambiental, promoção do enriquecimento ambiental, redução dos fatores estressantes que possam afetar o animal. Ademais, essa enfermidade exerce um impacto negativo sobre o abandono animal, pois animais com a Síndrome tendem a sofrerem abandono. Portanto, os estudos sobre essa alteração são importantes para atualização de profissionais permitindo promover o bem-estar e melhor expectativa de vida dos seus pacientes, além disso, conscientizar os tutores sobre a doença.
\end{abstract}

Palavras-chave: Trato urinário inferior; Gato; Estresse.

\begin{abstract}
Pandora's Syndrome is a disorder in the lower urinary tract of felines, its pathogenesis is not fully understood, but it is known that there are predisposing factors to its appearance, such as: genetic, low water intake, environmental stress, obesity, life modern cat household and lack of physical activity. The objective of this work was to report the main information described about this condition, in addition to its impact on the organism in these animals. Thus, articles were searched in document, article and book indexing databases, such as Scielo, Google Scholar, Pub Med and Scopus in the last 11 years, seeking more relevant and recent information about the disease. The syndrome presents
\end{abstract}


itself in a complex form that presents nonspecific clinical signs, making its diagnosis very difficult, requiring the use of several laboratory tests. Treatment involves multimodal therapy with environmental management, promotion of environmental enrichment, reduction of stressors that may affect the animal. Furthermore, this disease has a negative impact on animal abandonment, as animals with the Syndrome tend to suffer abandonment. Therefore, studies on this change are important for updating professionals, allowing them to promote the well-being and better life expectancy of their patients, in addition to making tutors aware of the disease.

Keywords: Lower urinary tract; Cat; Stress.

\section{Resumen}

El Síndrome de Pandora es un trastorno en el tracto urinario inferior de los felinos, su patogenia no se comprende del todo, pero se sabe que existen factores predisponentes a su aparición, tales como: genética, baja ingesta de agua, estrés ambiental, obesidad, vida gato moderno. hogar y falta de actividad física. El objetivo de este trabajo fue reportar la principal información descrita sobre esta condición, además de su impacto sobre el organismo en estos animales. Así, se realizaron búsquedas de artículos en bases de datos de indexación de documentos, artículos y libros, como Scielo, Google Scholar, Pub Med y Scopus en los últimos 11 años, buscando información más relevante y reciente sobre la enfermedad. El síndrome se presenta en una forma compleja que presenta signos clínicos inespecíficos, lo que dificulta su diagnóstico, requiriendo el uso de varias pruebas de laboratorio. El tratamiento implica terapia multimodal con manejo ambiental, promoción del enriquecimiento ambiental, reducción de factores estresantes que puedan afectar al animal. Además, esta enfermedad tiene un impacto negativo en el abandono de los animales, ya que los animales con el Síndrome tienden a sufrir el abandono. Por tanto, los estudios sobre este cambio son importantes para la actualización de los profesionales, permitiéndoles promover el bienestar y una mejor esperanza de vida de sus pacientes, además de concienciar a los tutores de la enfermedad.

Palabras clave: Tracto urinario inferior; Gato; Estrés.

\section{Introdução}

O termo Síndrome de Pandora é proposto para descrever casos em que gatos apresentam sinais clínicos do trato urinário inferior crônicos e recorrentes, na presença de desordens comportamentais, dermatológicas, endócrinas, gastrointestinais, dentre outras (Buffington et al., 2014). O termo parece apropriado por pelo menos duas razões. Primeiro, por não identificar qual a causa ou órgão específico e, segundo, por compreender os desafios associados à identificação de tantos problemas (Buffington, 2011).

A doença do trato urinário inferior dos felinos (DTUIF) abrange qualquer desordem que afeta a vesícula urinária ou a uretra dos gatos, que têm sinais clínicos inespecíficos para diagnóstico de uma dessas afecções (Nelson \& Couto, 2015). Atualmente, sugere-se que muitos dos casos que há presença de sinais clínicos crônicos e idiopáticos de DTUIF são diagnosticados com cistite idiopática felina (CIF), e podem, na realidade, ser Síndrome de Pandora (Buffington, 2011).

Os animais podem apresentar os seguintes sinais clínicos: hematúria, periúria, estrangúria, polaciúria, de forma aguda, crônica ou intermitente, cistite ulcerativa ou não ulcerativa, além de alterações em outros sistemas orgânicos (Luz, 2019). A DTUIF é uma razão comum para que tutores de gatos procurem por cuidados veterinários (Dorsch et al., 2014) em decorrência da expressão dos sinais clínicos nesses animais, entre eles, vocalização durante o ato miccional ou a dificuldade na hora da micção (Buffington et al., 2014).

Ademais, essa afecção apresenta sintomatologia inespecífica, dificultando o seu diagnóstico (Silva et al., 2013) e possui caráter recidivante, ou seja, o animal volta a apresentar os sinais clínicos da doença com certa frequência, após um intervalo de tempo (Teixeira et al., 2019). Portanto, esta revisão bibliográfica tem como objetivo levantar as principais informações descritas sobre essa afecção e possibilitar a atualização de profisssionais da área, a fim de promover o bem-estar e maior expectativa de vida dos felinos. Além disso, promover a conscientização dos tutores sobre a doença que tem importante impacto social.

\section{Metodologia}


Foi realizada uma revisão de literatura por meio de uma pesquisa bibliográfica a respeito da síndrome de Pandora, sobre suas principais descobertas ao longo dos últimos anos, como seu mecanismo de ação, sinais clínicos, diagnóstico e tratamento, em diferentes bases de indexação de documentos, artigos e livros, como Scielo, Google Scholar, Pub Med e Scopus, comparando essas diferentes informações sobre as descobertas dessa síndrome. Os critérios de inclusão obtidos através da leitura de artigos, livros, trabalhos de conclusão de curso e de mestrado publicados nos últimos 11 anos. Foram utilizadas as palavras-chave "síndrome de pandora", "DTUIF", "estresse em felinos" e "cistite idiopática felina".

\section{Resultados e Discussão}

O termo "Síndrome de Pandora" é utilizado para descrever gatos que apresentam recorrência crônica de sinais clínicos da doença do trato urinário inferior felino (DTUIF) de forma idiopática, concomitante a presença do acometimento de outros órgãos e sistemas, como, trato gastrointestinal, pele, pulmão, sistema cardiovascular, nervoso central, endócrino e sistemas imunológicos. Além da presença de sinais clínicos associados ao estresse (Buffington, 2011).

Essa síndrome é uma desordem multifatorial que pode acometer todas as idades, sexos e raças, entretanto ela acomete majoritariamente o sexo masculino entre dois à sete anos de idade. Pode estar interligada a fatores genéticos e a vida moderna do gato doméstico, através do manejo incorreto, da falta de atividades físicas, da baixa ingestão hídrica, da disponibilidade exclusiva de ração seca e do convívio inadequado com outros animais na residência. Pode gerar desordens como aumento do peso corporal, predispondo à obesidade, aumento da susceptibilidade a problemas no trato urinário inferior e o aumento gradativo do estresse, que pode levar ao desenvolvimento de doenças crônicas (Justen \& Santos, 2018; Teixeira et al., 2019).

\subsection{Mecanismos geradores da síndrome de Pandora}

A DTUIF ocorre por alterações na bexiga urinária e uretra, como urolitíase, tampões uretrais e defeitos anatômicos vesicais, que não possuem uma única causa (Lacerda et al., 2017). A Cistite Idiopática Felina (CIF) é o diagnóstico mais comum em gatos com DTUIF, ocorre em aproximadamente em 50 a 60\% dos casos (Weissova \& Norsworthy, 2011). Os nomes Doença do Trato Urinário Inferior Felino e Cistite Idiopática Felina tornaram-se limitados, visto que podem ser identificadas outras alterações nos felinos, como modificações comportamentais, endócrinas e dermatológicas (Buffington et al., 2014). Assim, Buffington (2011), após estudar esses felinos, sugeriu que fosse utilizado o termo Síndrome de Pandora para caracterizar essas alterações, pois não identifica nenhuma causa ou órgão específico.

A cistite idiopática felina é uma doença inflamatória, crônica, não infecciosa e de etiopatogenia ainda desconhecida. Seus sinais clínicos expressam-se em situações pós-estresse e dor, que gera um estímulo a liberação de cortisol pelo eixo hipotálamo-pituitária-adrenal e a ativação do sistema nervoso simpático, estimulando a liberação de catecolaminas, principalmente noradrenalina (Justen \& Santos, 2018). O processo inflamatório neurogênico ocorre através do estímulo de fibras C aferentes, liberação de neuropeptídeos, como a substância $\mathrm{P}$, leva a inflamação da vesícula urinária, deste modo, aumenta a permeabilidade vesical e possibilita o contato de agentes nocivos com as fibras aferentes sensoriais, que aumenta a inflamação e dor (Little, 2015).

A superfície interna do trato urinário inferior é revestida por uma camada de glicosaminoglicanos (GAG) e a vesícula urinária é revestida por um tipo específico de GAG, chamado GP-51. Fatores como pH alterado, concentração de eletrólitos, estimulação mecânica, química, agentes infecciosos, alterações neurais ou endócrinas podem prejudicar a integridade da barreira causando cistite (Buffington, 2011). Em gatos com CIF a camada de glicosaminoglicano (GAG) está diminuída, porém esta camada é essencial para a proteção do urotélio e prevenção da passagem da urina através desta (Little, 2015). Se a camada de GAG da vesícula urinária está comprometida, os componentes da urina (hidrogênio, cálcio e potássio) estimulam os neurônios sensitivos e tornam suscetível a uma inflamação na vesícula e, consequentemente, a estimulação da inflamação 
neurogênica é ativada. Ainda, ocorre um aumento da absorção intravesical de potássio, que consegue entrar em contato com neurônios aferentes e causar sua inibição, gerando assim retenção de urina na vesícula urinária, piorando o quadro clínico do animal (Vieira et al., 2017; Siqueira, 2020).

A inflamação neurogênica é um processo iniciado pela estimulação dos neurônios aferentes sensoriais da fibra C que, quando ativados, liberam neuropeptídeos (Justen \& Santos, 2018). Outras moléculas sinalizadoras oriundas de outros sistemas, como acetilcolina, substância $\mathrm{P}$ e prostaglandinas, também promovem a ativação de neurônios sensoriais aferentes e mastócitos. A presença de mastócitos causada principalmente por estresse foi encontrada em biópsias da vesícula urinária em felinos com CIF. A ativação dos mastócitos promove a liberação de inúmeras moléculas ativadoras responsáveis pela inflamação, vasodilatação, dor, fibrose e contração da musculatura lisa, o que exacerba os efeitos das fibras C. Um número crescente de mastócitos foi observado em cerca de $20 \%$ dos pacientes com CIF, e as pesquisas indicam que eles estão envolvidos na patofisiologia da síndrome (Buffington, 2011; Vieira et al., 2017).

Também foi identificado que alguns gatos com CIF possuem um sistema nervoso simpático mais sensível e hiperativo, causando aumento da enzima tirosina hidroxilase, principalmente na região do locus coeruleus e no núcleo paraventricular do hipotálamo, cuja função é limitar a velocidade de formação das catecolaminas. O locus coeruleus contém muitos neurônios noradrenérgicos e é a fonte mais importante de norepinefrina, um neurotransmissor responsável por ações de vigilância, excitação e analgesia, que parece mediar as respostas viscerais ao estresse (Buffington et al., 2014; Klein, 2014; Luz, 2019).

Ademais, o estresse também pode influenciar no mecanismo de resposta do eixo hipotálamo-hipófise-adrenal (HHA) com estimulação contínua do eixo. Sem resposta de aumento aos níveis plasmáticos de cortisol, não ocorre o feedback negativo pelos glicocorticóides, que são responsáveis por controlar a resposta ao estresse, bloqueando a sensação de dor transmitida ao cérebro e inibindo a libertação de mais catecolaminas (Alho, 2012; Justen \& Santos, 2018; Luz, 2019). Presumiu-se que o reforço da atividade do sistema nervoso simpático aumenta a permeabilidade do urotélio, e consequentemente, o aumento da atividade dos neurônios aferentes e nos sinais clínicos típicos da CIF (Vieira et al., 2017).

O mecanismo da síndrome de pandora é objeto de estudo a fim de maior compreensão, entretanto, as literaturas citam que o psicológico do animal está diretamente ligado com a origem da doença. O manejo do estresse é a peça-chave para o tratamento sintomático do felino acometido. A cistite intersticial ou bexiga dolorosa, que acomete humanos, principalmente mulheres acima de 40 anos, possui um conjunto de sinais clínicos idênticos aos que ocorrem na Síndrome de Pandora. Acredita-se que a ocorrência da doença em mulheres majoritariamente nesta faixa etária se dá em decorrência da demora para se alcançar o diagnóstico que ocorre a partir da exclusão de outros relacionados ao trato urinário inferior e apresenta curso crônico (Kraychete et al., 2017). Tal como na doença urinária felina, na grande maioria dos pacientes humanos, a etiologia da inflamação das vias urinárias é desconhecida (Freitas, 2014).

\subsection{Sinais clínicos}

Os sinais clínicos observados na Síndrome de Pandora são inespecíficos, já que se manifestam em todas as desordens do sistema urinário, dificultando assim o diagnóstico. Sinais como polaciúria, disúria, estrangúria, periúria, hematúria e vocalização durante o ato miccional, podendo ou não apresentar um quadro de obstrução, são os mais comumente encontrados (Silva et al., 2013), especialmente em gatos acometidos por DTUIFs (Buffington et al., 2014). Evidências indicam que animais acometidos pela Síndrome não lidam bem com o estresse, somando-se as alterações comportamentais e os fatores já citados que culminam no agravamento do quadro clínico (Alho, 2012). 
Outros sintomas relatados são mudança de humor, vômitos, anorexia, diminuição da ingestão de água, diminuição de interações sociais (Stella et al., 2011), bem como alopecia ventral abdominal e inguinal bilateral pelo excesso de autolimpeza crônica pela dor abdominal (Justen \& Santos, 2018).

O estilo de vida confinado, ao mesmo tempo que previne riscos como predação, traumatismos e contágio de doenças infecciosas, pode inibir alguns comportamentos naturais dos felinos e causar estresse. A resposta de estresse comportamental vem acompanhada por alterações imunológicas, neurológicas, endócrinas e vasculares (Little, 2015). Acredita-se que alguns gatos, pela associação entre genética e ambiente, possuem um sistema nervoso simpático mais sensível e hiperativo, e é considerado que esteja associado com as variações nos sistemas orgânicos do animal. Essas comorbidades podem ocorrer em qualquer combinação, e algumas delas podem preceder o desenvolvimento das doenças do trato urinário inferior. Esses distúrbios, assim como os estresses ambientais, podem manifestar-se como o que chamamos de "comportamentos de doenças (sick behaviors)", comportamentos que se referem a combinações variáveis de sinais clínicos e comportamentais. Os comportamentos de doença podem resultar das vias periférica (aferente) e central (eferente) (Buffington, 2011; Buffington, 2012).

\subsection{Diagnóstico}

O médico veterinário deve reconhecer que a coleção de sinais clínicos de DTUIF não serve como um diagnóstico definitivo (Robertson, 2014). Para um diagnóstico preliminar da síndrome, o clínico deve basear-se, principalmente, na análise minuciosa da história clínica e nos achados de exame físico (Buffington, 2019).

A anamnese deve ser voltada para a identificação dos sinais de trato urinário inferior, possíveis fatores de estresse e condições ambientais (Nelson \& Couto, 2015). O exame físico deve incluir a palpação da bexiga e a identificação de possível obstrução, podendo ser necessária a realização da desobstrução uretral (Santos, 2014). Caso haja obstrução uretral e sinais sistêmicos, a realização de hemograma e exames bioquímicos são recomendados para avaliar alterações hematológicas no animal, os níveis de uréia, creatinina e mensurar eletrólitos como potássio e fósforo. Se possível, também deve-se realizar a hemogasometria (Rodríguez, 2012). Para descartar outras patologias semelhantes à síndrome, mais exames devem ser realizados, tais como: urinálise e cultura de urina, exames de imagem (ultrassonografia e radiografia contrastada) para avaliar a presença de urólitos, infecção bacteriana, neoplasias e defeitos anatômicos (Crivellenti \& Crivellenti, 2015; Piyarungsri et al., 2020).

$\mathrm{Na}$ busca por formas de diagnósticos mais precisos, os pesquisadores dedicam-se à compreensão da doença. Buffington et al. (2014) realizaram um levantamento e desenvolveram alguns critérios provisórios que podem ser avaliados para o diagnóstico da síndrome. Primeiramente deve-se investigar a evidência de sinais clínicos de outros sistemas (sinais gastrointestinais, dermatológicos, respiratórios, endócrinos, comportamentais, em sistema nervoso ou imune). Na anamnese o clínico deve questionar se o felino em questão passou por experiências adversas precocemente, como a orfandade e o abandono. Outra informação a ser colhida na anamnese é se o aumento e diminuição dos sinais clínicos está associado a eventos que estimulem o estresse a nível central. O último dos critérios elaborados, é se a resolução dos sinais clínicos está associada à modificação ambiental multimodal eficaz. Tais critérios podem ser utilizados enquanto métodos mais assertivos de diagnóstico não são desenvolvidos.

\subsection{Tratamento}

Em face da etiologia desconhecida da CIF e da recorrência de episódios de crise desta enfermidade, o tratamento direciona-se para a redução da frequência e da gravidade em que ocorrem os quadros clínicos (Little, 2015). Na maioria dos 
casos, a doença é autolimitante, resolvendo-se em cinco a sete dias com ou sem tratamento, entretanto, a terapêutica é recomendada, pois seu caráter doloroso e estressante pode implicar o aparecimento de outras afecções como obstrução uretral, alterações comportamentais e autolesões na região perineal (Reche \& Camozzi, 2015).

Existe uma variabilidade individual em relação aos aspectos que podem ser estressantes aos felinos, de forma que o manejo dessa enfermidade depende de diversos fatores como a presença ou não de obstrução, a identificação do episódio como inicial ou recidiva e os sinais clínicos daquele animal. Assim, cada animal deve ser avaliado individualmente, como também sua terapia deve ser voltada para seu quadro clínico (Reche \& Camozzi, 2015).

O tratamento envolve a redução dos agentes causadores de estresse ao felino, haja visto que, conforme ocorre uma hiperexcitação simpática, a resposta inflamatória crônica se mantém de forma responsiva a esse estímulo (Little, 2015). Assim, a prevenção é essencial, no qual o proprietário deve estar atento às manifestações prodrômicas e reconhecer atividades que sejam estressantes ao felino, como alterações ambientais ou a presença de outros animais no mesmo ambiente (Reche \& Camozzi, 2015). Tais fatores ressaltam a importância da relação do médico veterinário com o responsável, já que assegurar a compreensão do proprietário em relação à CIF previne o atraso da identificação da doença, garantindo um prognóstico favorável ao paciente e a redução da ocorrência de recidivas.

Os três principais pilares do tratamento da CIF consistem na redução do estresse, na terapêutica medicamentosa e na alteração da dieta do felino, que passa a ser de alimentos mais úmidos e pastosos, com o objetivo de aumentar a ingestão hídrica que resulta na redução da densidade urinária, diminuindo, consequentemente, a concentração de propriedades nocivas da urina que irritam o urotélio fragilizado (Forrester \& Towell, 2015; Oliveira et al., 2017).

Outros cuidados recomendados para atingir um bom prognóstico incluem a modificação ambiental multimodal (MEMO - "Multimodal environmental modifications"), uma abordagem multidisciplinar a qual atesta que o enriquecimento ambiental, realizada como terapia preventiva, a qual beneficia os gatos que vivem restritos em domicílio, trazendo melhorias à sua saúde, além do bem-estar físico e psicológico na maioria dos casos, por reduzir a probabilidade de ativação do sistema de resposta ao estresse do felino (Buffington et al., 2014). Desse modo, esta terapia reduz a frequência de episódios de CIF e a gravidade com que ocorrem. Tais mudanças incluem, portanto, a educação do tutor, já que a relação do responsável com o animal favorece a socialização do animal e reduz o estresse; também inclui variáveis mudanças no ambiente físico e na dieta do gato, como o manejo nutricional, que passa a ser baseado em uma dieta úmida, oferecida de forma interativa para estimular a caça, como a fonte de bebidas, de forma que o manejo instituído estimule uma maior ingestão de água pelo animal; higienização da caixa sanitária sempre que possível e situada em local privado e silencioso, com substrato de preferência do animal; manejo de áreas de entradas e saída da habitação e áreas de descanso e entretenimento (Vieira et al., 2017).

Outros tratamentos alternativos têm sido relatados em estudos como modalidades de enriquecimento ambiental, como o uso de feromônios sintéticos, pois atuam a nível de hipotálamo e do sistema límbico, modificando o estado emocional do animal, sendo, portanto, capazes de reduzir o estresse e brigas entre animais nas residências. Entretanto, revisões recentes afirmam não ter evidências de que seu uso, de fato, diminui o estresse e acalma felinos (Vieira et al., 2017); práticas de medicina alternativa também têm sido relatadas, como as práticas de acupuntura para redução de estresse no tratamento da CIF (Teixeira et al., 2019), ademais, deve-se atentar ao fato de que tais terapias devem ser adaptadas a cada caso em particular mediante às características comportamentais, emocionais e médicas de cada animal.

A terapia medicamentosa para CIF é recomendada apenas em casos graves ou recidivantes, principalmente quando se trata de medicação oral, visto que sua administração é estressante à maioria dos felinos. Nos casos em que o enriquecimento ambiental e a alteração da dieta não são satisfatórios, recomenda-se o uso de antidepressivos tricíclicos como a amitriptilina, com dose variando de 2,5 a 12,5 mg/gato a cada 24 horas, quando administrada por via oral, dado sua propriedade anticolinérgica, anti-histamínica, simpaticolítica, anti-inflamatória e analgésica (Reche \& Camozzi, 2015). A dosagem deve ser 
aumentada gradativamente conforme a sintomatologia do animal se manifesta, durante um período de quatro meses, e descontinuada caso não haja resultados satisfatórios após este período (Vieira et al., 2017). A amitriptilina não é recomendada em casos agudos, sendo seus benefícios constatados a longo prazo (Little, 2015). Outros analgésicos como buprenorfina, adesivo transdérmico de fentanila, butorfanol, oximorfona, fentanila, e anti-inflamatórios não esteroidais são recomendados, atentando-se sempre para a via de administração, que deve ser sempre fácil para evitar o estresse do animal (Little, 2015).

A terapia com glicosaminas (Terapia GAG), consiste em estimular a recuperação do urotélio através do uso desta classe de mucopolissacarídeos de características hidrorrepelentes, analgésica e anti-inflamatória, que atuam como substrato natural para a biossíntese de GAG (Little, 2015). A glicosamina é acessível, geralmente associada a sulfato de condroitina, no entanto, a eficácia de sua utilização ainda não está comprovada cientificamente quando se trata da terapia para CIF (Reche \& Camozzi, 2015).

Dessa forma, a terapêutica farmacológica é apenas paliativa, portanto as medidas que visam a redução do estresse do animal são as mais satisfatórias (Vieira et al., 2017). Quando a investigação diagnóstica exclui outras causas da CIF, o prognóstico se torna favorável, desde que seja realizado o tratamento multimodal e com a instrução do tutor acerca da identificação da enfermidade previamente, para que não haja comprometimento na recuperação do paciente e o prolongamento de seu sofrimento (Weissova \& Norsworthy, 2011).

A Síndrome de Pandora possui impacto ético-social, devido aos sintomas de periúria e recidivas constantes, levando o tutor a considerar o abandono e até mesmo a eutanásia do animal (Fernandes, 2017). A observação repetida de que muitos desses problemas e comportamentos são resolvidos após a exposição a um ambiente enriquecido, fornece evidências adicionais de um distúrbio do sistema nervoso central resultante em uma doença multissistêmica crônica, afetando de forma variável a bexiga e outros órgãos (Stella et al., 2011).

\section{Considerações Finais}

A Síndrome de Pandora apresenta uma fisiopatologia complexa e pouco esclarecida. Acomete o trato urinário inferior desenvolvendo uma sintomatologia inespecífica que é refletida em diversos órgãos, sendo uma característica marcante a obstrução urinária frequente. O principal fator que contribui para o seu surgimento é o estresse em que o felino é exposto estando estreitamente interligado com o seu prognóstico. Seu diagnóstico é complexo e eliminatório, devendo sempre excluir outras causas bases (outras doenças urinárias) para sua confirmação, assim, torna sua identificação mais difícil. Tendo em vista a não existência de cura para essa enfermidade, às modalidades terapêuticas utilizadas têm um carácter paliativo, a fim de aliviar válvulas de estresse e controle de dor. Além do enriquecimento ambiental, uma peça-chave de prevenção e tratamento dessa patologia.

\section{Referências}

Alho, A. M. P. V. A. (2012). O enriquecimento ambiental como estratégia de tratamento e prevenção da cistite idiopática felina. [Dissertação de Mestrado, Universidade Técnica de Lisboa]. Repositório da Universidade de Lisboa. https://www.repository.utl.pt/handle/10400.5/4064

Buffington C. A. (2011). Idiopathic cystitis in domestic cats-beyond the lower urinary tract. Journal of veterinary internal medicine, 25(4), 784-796. https://doi.org/10.1111/j.1939-1676.2011.0732.x.

Buffington, C. A. T. (2012). Síndrome de Pandora: Reconsiderando nuestro enfoque a la cistitis idiopática en gatos. Veterinary Medicine en Español, 6(6), 513.

Buffington, С. А. Т. (2019). Синдром Пандоры у кошек. Диагностика, терапия https:/vk.com/@vet_news-sindrom-pandory-u-koshek-diagnostikaterapiya

Buffington, C. A. T., Westropp, J. L., \& Chew, D. J. (2014). From FUS to Pandora syndrome: where are we, how did we get here, and where to now?. Journal of feline medicine and surgery, 16(5), 385-394. https://doi.org/10.1177/1098612X14530212

Crivellenti, L. Z \& Crivellenti, S. B. (2015). Casos de rotina em medicina veterinária de pequenos animais ( $2^{\mathrm{a}}$ ed.). Medvet. 
Dorsch, R., Remer, C., Sauter-Louis, C., \& Hartmann, K. (2014). Feline lower urinary tract disease in a German cat population: A retrospective analysis of demographic data, causes and clinical signs. Tierärztliche Praxis Kleintiere, 42(4), 231-239.

Fernandes, C M S. (2017). Síndrome de pandora: prevenção e tratamento: revisão sistemática. [Trabalho de conclusão de curso, Universidade Estadual Paulista Julio de Mesquita Filhos]. Repositório Institucional da UNESP. http://hdl.handle.net/11449/156706.

Forrester, S. D., \& Towell, T. L. (2015) Feline idiopathic Cystitis. Veterinary Clinics of North America: Small Animal Practice, $45(4), 783-806$.

Freitas, L. S. M. (2014). Diagnósticos diferenciais da Cistite Intersticial/Síndrome Doloroso Vesical. [Dissertação de Mestrado, Universidade do Porto]. Repositório Aberto da Universidade do Porto. https://hdl.handle.net/10216/72872

Justen, H., \& Santos, C.R.G. (2018). Cistite idiopática felina: aspectos clínicos, fisiopatológicos e terapêuticos. Boletim PET, v.1.

Klein, B. G. (2014). Cunningham tratado de fisiologia veterinária (5 ed.). Rio de Janeiro: Elsevier.

Kraychete, D. C., Siqueira, J. T. T., Garcia, J. B., Sakata, R. K., Sousa, Â. M., Andrade, D. C., Zakka, T. R. M., \& Teixeira, M. J. (2017). Clinical evidence on visceral pain. Systematic review. Revista Dor, 18(1), 65-71. https://doi.org/10.5935/1806-0013.20170014

Lacerda, L., Grein, J. M., Vanhoni, M. S., \& Scheraiber, M. (2017). Doença do Trato Urinário Inferior dos Felinos - Relato de Caso. Revista eletrônica Biociências, Biotecnologia e Saúde, 10(19), 94-96.

Little, S. E. (2015). O gato: medicina interna. (1 ${ }^{\mathrm{a}}$. ed.) Rio de Janeiro: Roca

Luz, A. C. G. (2019). Síndrome de pandora e caminhos para investigação clínica. [Trabalho de conclusão de curso, Universidade Federal Rural da Amazônia]. Biblioteca Digital de Trabalhos Acadêmicos. http://bdta.ufra.edu.br/jspui/handle/123456789/1000

Nelson, R. W. \& Couto, C. G. (2015). Medicina interna de pequenos animais. (5 a ed.) Guanabara Koogan.

Oliveira, M. R. B., Silva, C. R. A., Jesus, K. C. D., Rodrigues, K. F., Silva, R. A., Costa, S. D. P., Silva, F. L., \& Rodrigues, M. C. (2017). Diagnosticando a cistite idiopática felina. Revisão. Medicina Veterinária e Zootecnia, 11(9), 864-876. http://dx.doi.org/10.22256/PUBVET.V11N9.864-876

Piyarungsri, K., Tangtrongsup, S., Thitaram, N., Lekklar, P., \& Kittinuntasilp, A. (2020). Prevalence and risk factors of feline lower urinary tract disease in Chiang Mai, Thailand. Scientific Reports, 10(1), 196. https://doi.org/10.1038/s41598-019-56968-w.

Reche Junior, A., \& Camozzi, R. B. (2015). Doença do Trato Urinário Inferior dos Felinos: Cistite Intersticial. In: Jericó, M. M., Neto, J. P. A., \& Kogica, M. M. Tratado de medicina interna de cães e gatos. (pp. 1483-1492). Roca.

Robertson, E. (2014). Feline cystitis: a case presenting with LUTS in a young female cat. Companion Animal, 19(6), $284-287$. https://doi.org/10.12968/coan.2014.19.6.284

Rodriguez, Ó. C. (2012). Manual de nefrología y urología clínica canina y felina (2 ${ }^{\mathrm{a}}$ ed). Servet.

Santos, K. K. F. (2014). Guia prático de nefrologia em cães e gatos (1 ${ }^{\mathrm{a}}$ ed.). L. F. Livros.

Silva, A. C., Muzzi, R. A. L., Oberlender, G., Muzzi, L. A. L., Coelho, M. de R., \& Henrique, B. F. (2013). Cistite idiopática felina: revisão de literatura. Arq. Ciênc. Vet. Zool. UNIPAR, 16(1), 93-96.

Siqueira, T. de S. (2020) Doença do trato urinário inferior dos felinos e suas implicações sistêmicas: revisão de literatura. [Trabalho de conclusão de curso, Universidade Federal da Paraíba]. Repositório Institucional da UFPB. https://repositorio.ufpb.br/jspui/handle/123456789/19232

Stella, J. L., Lord, L. K., \& Buffington, C. A. (2011). Sickness behaviors in response to unusual external events in healthy cats and cats with feline interstitial cystitis. Journal of the American Veterinary Medical Association, 238(1), 67-73. https://doi.org/10.2460/javma.238.1.67

Teixeira, K. C., Vieira, M. Z., \& Torres, M. L. M. (2019). Síndrome de Pandora: aspectos psiconeuroendócrinos. Revista de Educação Continuada em Medicina Veterinária e Zootecnia do CRMV-SP, 17(1), 16-19.

Vieira, A. N. L. S., Ramos, P. R. R., Melchert, A., \& Guimarães-Okamoto, P. T. C. (2017). Feline Pandora's Syndrome: a bibliographic review. Veterinária e Zootecnia, 24(4), 680-690. https://doi.org/10.35172/rvz.2017.v24.239

Weissova, T., \& Norsworthy, G.D. (2011). Feline idiopathic cystitis. In: Norsworthy, G. (Eds.), The feline patient (pp.176-178). Wiley-Blackwell. 